\title{
Viral Taxonomy
}

Marjorie J. Miller

From the Department of Pathology and Laboratory Medicine,

UCLA Medical Center, Los Angeles, California

$\begin{array}{lll}\text { Family Subfamily } & \text { Genus } & \text { (No. of Members) }\end{array}$

\section{RNA Viruses}

Picornaviridae

Caliciviridae

Reoviridae

Togaviridae

Flaviviridae

Rubivirus

Heparnavirus

Rhinovirus

Calicivirus

Hepevirus

Orbivirus

Rotavirus

Alphavirus

Flavivirus

Hepacavirus

Orthomyxoviridae

Paramyxoviridae

Influenzavirus

Paramyxovirus

Morbillivirus

Pneumovirus

Rhabdoviridae

Filoviridae

Coronaviridae
Lyssavirus

Filovirus

Coronavirus
Polioviruses (3)

Coxsackie A viruses (23)

Coxsackie B viruses (6)

Echoviruses (31)

Enteroviruses 68-71 (4)

Hepatitis A virus (enterovirus 72) (1)

Common cold viruses $(>115)$

Norwalk and Norwalk-like gastroenteritis viruses

(many)

Hepatitis E virus (enterically transmitted non-A, non-B hepatitis) (1)

Colorado tick fever (1) and Kemerovo viruses (1)

Human rotaviruses $(>6)$

Western, Eastern, and Venezuelan equine encephalitis viruses, Sindbis and Semliki Forest viruses (group A arboviruses, mosquito-borne viruses) (23)

Rubella virus (1)

St. Louis, Japanese B, and Murray Valley encephalitis viruses; yellow fever, dengue, and West Nile fever viruses (group B arboviruses, mosquito-borne viruses) (26)

Kyasanur Forest disease, Omsk hemorrhagic fever, European and Far Eastern tick-borne encephalitis (tick-borne viruses) (11)

Vector-unassociated viruses (17)

Hepatitis $C$ virus (parenterally transmitted non-A, non-B hepatitis)

Influenza virus types A (many), B (several) and C (1)

Parainfluenza viruses (4)

Mumps virus (1)

Measles virus (1)

Respiratory syncytial virus (1)

Rabies virus (1)

Marburg (1) and Ebola viruses (2)

Human coronaviruses (2)

Correspondence: Dr. Marjorie J. Miller, UCLA Clinical Laboratories, 11633 San Vicente Boulevard, Fourth floor, Los Angeles, California 90049. 
Family

Subfamily

Genus

RNA Viruses (Continued)

Bunyaviridae

Arenaviridae

Lentivirinae

Phlebovirus

Nairovirus

Uukuvirus

Hantavirus

Type $\mathrm{C}$ oncovirus

Lentivirus

Arenavirus

\section{Species Pathogenic}

for Humans

(No. of Members)

$\begin{array}{lll}\text { Bunyaviridae } & \text { Bunyavirus } \\ & \\ & \begin{array}{l}\text { Phlebovirus } \\ \text { Nairovirus }\end{array} \\ & \begin{array}{l}\text { Uukuvirus } \\ \text { Hantavirus }\end{array} \\ \text { Retroviridae } & \text { Oncovirinae } & \text { Type C oncovirus } \\ & \text { Lentivirinae } & \text { Lentivirus } \\ \text { Arenaviridae } & & \text { Arenavirus }\end{array}$

DNA Viruses

Parvoviridae

Papovaviridae

Adenoviridae Hepadnaviridae Herpesviridae

Poxviridae
Parvovirus

Papillomavirus

Polyomavirus

Mastadenovirus

Hepadnavirus

Alphaherpesvirinae

Betaherpesvirinae

Gammaherpesvirinae Chordopoxviridae
Simplexvirus

Varicellovirus

Cytomegalovirus

HHV-6

Lymphocryptovirus

Orthopoxvirus

Parapoxvirus

Yatapoxvirus

Molluscipoxvirus
Bunyamwera virus (Bunyamwera serogroup),

California encephalitis, and LaCrosse viruses

(California serogroup)

Viruses of other serogroups (>145)

Sandfly fever viruses, Rift Valley fever virus $(>30)$

Crimean-Congo hemorrhagic fever virus

Viruses of five other serogroups $(>27)$

Uukuniemi and other viruses (7)

Hantaan virus (Korean hemorrhagic fever or hemorrhagic fever with renal syndrome) (many)

Human T-cell leukemia-associated virus types I and II $(>2)$

Human immunodeficiency viruses 1 and 2 (2)

Lymphocytic choriomeningitis virus (1)

Lassa fever virus (I)

Junin (Argentine hemorrhagic fever virus)

Machupo (Bolivian hemorrhagic fever virus)

Human parvovirus B 19 (erythema infectiosum, or fifth disease) and RA-l ( $>2)$

Papilloma viruses (many)

$\mathrm{BK}$ and $\mathrm{JC}$ viruses (2)

Adenoviruses (42)

Hepatitis B virus (1)

Herpes simplex virus, types 1 and 2 (2)

Varicella-zoster virus (1)

Cytomegalovirus (1)

Human herpesvirus 6 (lymphotropic human herpesvirus) (1)

Epstein-Barr virus (1)

Vaccinia virus (1)

Smallpox virus (variola) (1)

Orf virus (?)

Virus of milker's nodule (1)

Yaba- and tanapox viruses (2)

Molluscum contagiosum virus (1) 